\title{
Genesis of Still's murmurs: a controlled Doppler echocardiographic study
}

\author{
H M Gardiner, H S Joffe
}

\begin{abstract}
Objective-The origin of innocent systolic murmurs is uncertain. This study was designed to evaluate the relative contribution in the same subjects of the main factors implicated in the genesis of vibratory innocent systolic murmursthat is, the presence of left ventricular bands and increased left ventricular flow indices-and to examine evidence for turbulence in relation to these findings.

Patients-40 children with innocent systolic murmurs and 40 paired controls without murmurs were matched for age and body surface area.
\end{abstract}

Design-These subjects were examined prospectively by cross sectional echocardiography with pulsed and continuous wave Doppler and colour flow mapping.

Setting-A tertiary referral centre for paediatric cardiology.

Results-Left ventricular bands were significantly more prevalent in patients with innocent murmurs $(70 \%)$ than in controls $(48 \%)$ but only if apical bands were included ( $p=0.039)$. Cases had significantly higher aortic peak flow velocities $(\mathbf{p}<0.001)$, flow integrals $(p=0.001)$, and stroke volumes $(p=0.014)$, and significantly lower heart rates $(p=0.033)$ than controls. There were no significant differences between cases and controls in mean aortic diameter, cardiac output, or ejection fraction. There was no evidence of turbulence in the left ventricle or outflow tract in any individual but curvilinear periodic vibrations and audible "whoops" were detected in the left ventricle by pulsed wave Doppler echocardiography. These were significantly more common in cases than controls ( $p<0.01)$ but were not related to the presence of bands.

Conclusions-Innocent systolic murmurs were unrelated to aortic size but they were strongly associated with higher aortic flow volume and velocity, with relative bradycardia, and with evidence of vibratory phenomena in the left ventricle. The role of left ventricular bands remains uncertain.

Sick Children, Bristo H M Gardiner

H S Joffe

Correspondence to

Dr H S Joffe, Royal Hospital for Sick Children, $\mathrm{St}$

Michael's Hill, Bristol BS2

8BJ.

Accepted for publication

Accepted for
Innocent systolic murmurs are audible in up to $70 \%$ of school-children. ${ }^{1}$ They are characterised by their musical sound and, as their name suggests, are benign and cause no symp- toms. They usually disappear during adolescence. The origin of a musical systolic murmur is still a matter for debate: some studies suggest that it is caused by the vibration of intracardiac structures such as valves in hearts with structural abnormalities ${ }^{2-4}$; others suggest that vibrations of left ventricular bands or turbulent flow about these structures cause the innocent systolic murmur. ${ }^{56}$

Left ventricular flow characteristics have also been investigated as a possible cause of the murmur. Schwarz et al reported that the peak velocities in the ascending and descending aorta were significantly greater in children with innocent murmurs and ascribed this to a narrower aortic diameter in this group compared with children without murmurs. ${ }^{7}$ These findings were confirmed by Sholler and colleagues $^{8}$ but not by Van Oort et al who found no difference in the peak velocity, acceleration, or spectral width of the aortic flow signal between children with innocent murmurs and children without. ${ }^{9}$ There is currently no consensus on the origin of the musical murmur in structurally normal hearts.

This study was designed to determine the prevalence of bands, the presence of turbulence about the bands and in the left ventricular outflow tract, and left ventricular flow characteristics and systolic function in children with innocent murmurs and without.

\section{Patients and methods}

We studied 40 symptom free children (aged 5 months to 13 years) who had been referred to the Bristol Children's Hospital for evaluation of murmurs and were found to have the characteristic musical and vibratory bruit of the Still's innocent murmur. The control group consisted of 40 children without murmurs. They were inpatients who were fully recovered after a transient acute illness $(40 \%)$ or children whose parents had responded to a written request for participants for this study $(60 \%)$. Each study child was matched for age and body surface area with a control child (table 1).

The children were studied in the supine or left decubitus position by the Hewlett Packard $77500 \mathrm{C}$ machine with a 5 or $3.5 \mathrm{MHz}$ transducer. A concurrent electrocardiograph recording was taken to time events and obtain heart rates. The heart was examined in standard planes by cross sectional real time ultrasound and pulsed and continuous wave Doppler. 
Table 1 Clinical and flow data (mean [SD ]) of cases and controls

\begin{tabular}{|c|c|c|c|c|}
\hline Data & $\begin{array}{l}\text { Cases } \\
(n=40)\end{array}$ & $\begin{array}{l}\text { Controls } \\
(n=40)\end{array}$ & $t$ test & pvalue \\
\hline $\begin{array}{l}\text { Age (month) } \\
\text { BSA (m²) } \\
\text { Aortic data: }\end{array}$ & $\begin{array}{l}60(39) \\
0.76(0.26)\end{array}$ & $\begin{array}{l}63(40) \\
0.77(0.26)\end{array}$ & $\begin{array}{l}1 \cdot 14 \\
0 \cdot 46\end{array}$ & $\begin{array}{l}\text { NS } \\
\text { NS }\end{array}$ \\
\hline $\begin{array}{l}\text { Ao diam }(\mathrm{cm}) \\
\text { Acceln }\left(\mathrm{m} / \mathrm{s}^{2}\right) \\
\text { Peak flow }(\mathrm{m} / \mathrm{s}) \\
\text { Flow integral } \\
\text { EF } \\
\text { Stroke vol (ml) } \\
\text { Heart rate (beats } / \mathrm{min}) \\
\text { CO }(1 / \mathrm{min})\end{array}$ & $\begin{array}{c}1.41(0.24) \\
23.38(4.96) \\
1.62(0 \cdot 18) \\
26.59(4.45) \\
0.81(0.07) \\
44 \cdot 12(18 \cdot 18) \\
94.8(23.5) \\
3.96(1.38)\end{array}$ & $\begin{array}{c}1.44(0.29) \\
21.70(5.58) \\
1.41(0.19) \\
21.20(3.39) \\
0.80(0.06) \\
38.07(18.39) \\
101.8(22.0) \\
3.62(1.27)\end{array}$ & $\begin{array}{l}0.72 \\
1.57 \\
5 \cdot 05 \\
6.05 \\
0 \cdot 28 \\
2.58 \\
2 \cdot 21 \\
1 \cdot 20\end{array}$ & $\begin{array}{l}\text { NS } \\
\text { NS } \\
<0.001 \\
<0.001 \\
\text { NS } \\
0.014 \\
0.033 \\
\text { NS }\end{array}$ \\
\hline $\begin{array}{l}\text { Pulmonary data: } \\
\text { Peak flow }(\mathrm{m} / \mathbf{s}) \\
\text { Flow integral }\end{array}$ & $\begin{array}{l}(n=28) \\
82.92(12 \cdot 86) \\
14.46(3 \cdot 19)\end{array}$ & $\begin{array}{l}(\mathrm{n}=28) \\
86.37(13.60) \\
15.65(2 \cdot 25)\end{array}$ & $\begin{array}{l}-0.64 \\
-1.22\end{array}$ & $\begin{array}{l}\text { NS } \\
\text { NS }\end{array}$ \\
\hline
\end{tabular}

BSA, body surface area; Ao diam, aortic diameter; Acceln, acceleration; EF, ejection fraction; Stroke vol, stroke volume; CO, cardiac output.

PREVALENCE OF LEFT VENTRICULAR BANDS

The number and site of any bands in the left ventricle was noted. Off axis views were sometimes required to determine the presence of bands and to distinguish them from left ventricular trabeculations. ${ }^{10}$

\section{ASSESSMENT OF TURBULENT FLOW}

The pulsed wave Doppler sample was placed at three sites along the apical aspect and at three similar sites along the basal aspect of the band and the flow pattern in each location was recorded. Similar recordings were made in the children without bands, by sampling the four sites nearest to the ventricular walls to enable the wave patterns produced about the bands to be compared with those produced by the trabeculae along the ventricular walls. Pulsed wave Doppler recordings were also made approximately $1 \mathrm{~cm}$ proximal to the aortic valve in the left ventricular outflow tract. Colour flow imaging of the left atrium, left ventricle, and left ventricular outflow tract was recorded from the parasternal and apical long axis views. A high parasternal long axis view was used to assess flow patterns in the aortic root and ascending aorta and this or a short axis view was used to record flow in the right ventricular outflow tract and pulmonary artery.

\section{LEFT VENTRICULAR FUNCTION}

Continuous wave Doppler recordings ${ }^{11}$ were made from the suprasternal notch ${ }^{12}$ and the maximum velocity (peak flow), time to peak (acceleration), and flow integral in the ascending aorta were measured in systole (the mean of six measurements for each index was recorded). We used a non-steerable off axis continuous wave pencil probe because this facilitated the acquisition of higher quality and more uniform traces in children of various sizes than the larger pulsed wave probe. ${ }^{14}$ Because continuous wave Doppler measures velocity within the aortic ring or the narrowest portion of the left ventricular outflow tract, ${ }^{15}$ the diameter of the aortic root was calculated from a mean of six measurements taken at the origin of the cusps during maximal separation of the aortic valve leaflets.

We used the Hewlett Packard software package to calculate the stroke volume from the flow integral and cross sectional area of the aorta and the cardiac output from the stroke volume and the heart rate. For the ejection fraction the internal diameter of the left ventricle was measured at the level of the tips of the mitral valve-firstly at the end of diastole (Ed) just after mitral valve closure and then at the end of systole (Es) just after aortic valve closure but before mitral valve opening. The ejection fraction was calculated as Ed - Es/ Ed.

\section{RIGHT VENTRICULAR FUNCTION}

Pulmonary peak velocities and flow integrals were recorded by pulsed wave Doppler from a high parasternal long or short axis view in all cases where adequate traces were obtained (28 pairs of children). Because of poor reproducibility of pulmonary artery diameters, ${ }^{16}$ derived estimates of right ventricular stroke volume and cardiac output were not obtained.

\section{DATA COLLECTION}

The examinations were recorded on videotape for later batch analysis by the Hewlett Packard software package. Data analysis was performed without knowledge of the status of the child (case or control).

\section{STATISTICAL ANALYSIS}

McNemar, $\chi^{2}$, and Student's $t$ test were used to analyse paired non-parametric data, nonpaired non-parametric data, and paired parametric data respectively. A $p$ value of $<0.05$ was regarded as significant.

\section{Results}

\section{PREVALENCE OF LEFT VENTRICULAR BANDS}

Twenty eight cases $(70 \%)$ and 19 controls $(48 \%)$ had bands in the left ventricle. Several subjects had more than one band and table 2 shows the number and location of bands. Bands of all types were significantly more common in children with murmurs than in those without (McNemar's test, $\mathrm{p}=0.0389$ ) and the relative risk of a child with an innocent murmur having a band in this study was calculated as $2 \cdot 8: 1$ (95\% CI $1 \cdot 2$ to $10 \cdot 1)$. However, if the four children with apical bands only were regarded as having no bands the difference between the two groups was not significant. Unlike the findings of Darazs et al, there was no significant difference in the location of the bands between the two groups in this study. ${ }^{6}$ 
Table 2 Number and location of left ventricular bands

\begin{tabular}{lccccc}
\hline & \multicolumn{2}{c}{ Number of children } & & \multicolumn{2}{c}{ Number of bands } \\
\cline { 2 - 5 } \cline { 5 - 6 } Site of band & Cases & Controls & & Cases & Controls \\
\hline LVOT & 2 & 2 & 2 & 2 \\
MC & 13 & 13 & 15 & 15 \\
A/S & 4 & 0 & 6 & 0 \\
LVOT + MC & 1 & 1 & 3 & 2 \\
LVOT + A/S & 5 & 0 & 12 & 0 \\
MC+A/S & 2. & 3 & 3 & 8 \\
LVOT + MC + A/S & 1 & 0 & 47 & 27 \\
Total & 28 & 19 & & \\
\hline
\end{tabular}

LVOT, left ventricular outflow tract; MC, mid-cavity; A, apex; S, septum.

ASSESSMENT OF TURBULENCE

By pulsed wave Doppler

Unusual Doppler patterns within the left ventricle were detected in 25 children (figure). These took the form of curvilinear harmonic vibrations during systole, usually in the opposite direction to the flow signal, and they were associated with audible simultaneous "whoops" on the microphone. These patterns were seen more often in cases than controls (18/ $\left.40 v 7 / 40 ; \chi^{2}=7.040, \mathrm{p}<0.01\right)$. The harmonic vibrations were seen about the bands in $19(38 \%)$ of the 47 children with bands and in seven $(21 \%)$ of the 33 children without bands-along the septum in two cases, in the left ventricular outflow tract in a further two cases, and at both sites in three. There was no significant difference in the frequency of curvilinear systolic vibrations between the children with bands and those without $\left(\chi^{2}=3 \cdot 262, p=N S\right)$ and there was no relation between these harmonic flow patterns and the position or orientation of the bands.

The 28 children with bands but no detectable evidence of turbulence included 15 who also had murmurs.

\section{By colour flow mapping}

We were unable to detect colour flow evidence of turbulent flow about the bands, along the ventricular walls, or in the left ventricular outflow tract in any of the cases or controls.

LEFT VENTRICULAR FLOW CHARACTERISTICS

Table 1 shows all the measurements (mean (SD)) and paired $t$ test analyses. There was no significant difference in the diameter of the

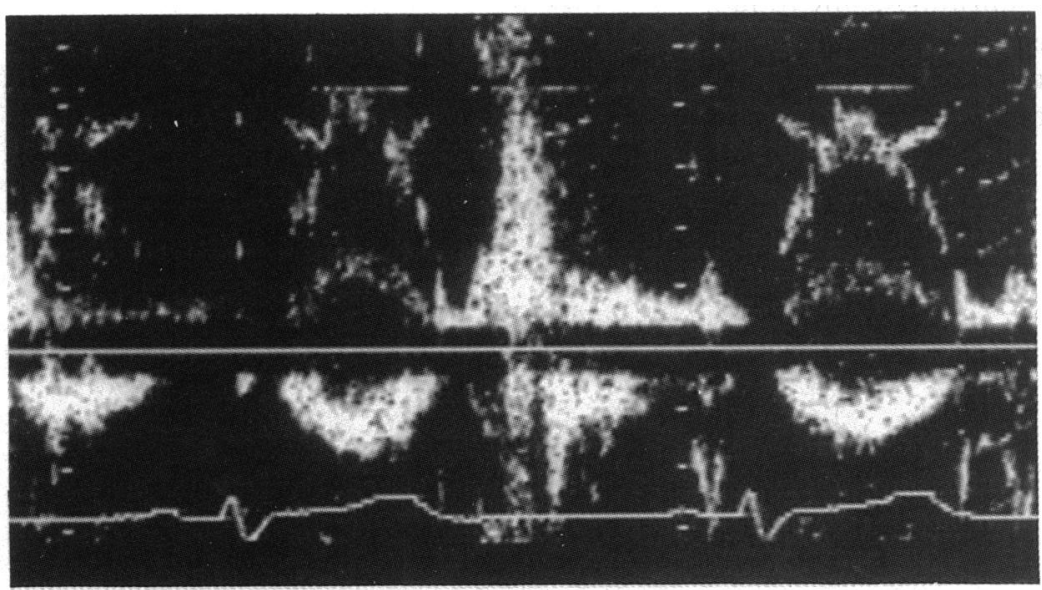

Pulsed wave Doppler as seen adjacent to trabeculae or about a left ventricular band, showing curvilinear periodic vibrations during systole in the opposite direction to the negative flow signal. aortic root between the cases and controls nor in the rate of acceleration of flow through the valve. The ejection fraction did not differ in the two groups of children; but the mean peak flow, flow integral, and stroke volume were significantly greater in the cases than the controls. However, the cardiac output of the cases was similar to that of the controls, mainly owing to the significantly lower heart rate in the cases.

\section{RIGHT VENTRICULAR FLOW CHARACTERISTICS}

There was no significant difference in the mean pulmonary peak flow or flow integrals between the cases and the controls.

\section{Discussion}

Van Oort et al stated that innocent murmurs could not commonly be produced by left ventricular bands because bands were much less prevalent $(0.8 \%$ paediatric patients) than innocent murmurs. ${ }^{9}$ However, the reported prevalence of left ventricular bands varied widely and often depended on whether the study was prospective or retrospective because off axis views were often essential to detect their presence. ${ }^{101718}$ The prevalence of bands in this study (70\% cases and $48 \%$ controls) was less than that reported in Darazs et al's prospective study ( $92 \%$ and $64 \%$ respectively), but they found a prevalence of only $22 \%$ in their first retrospective report. ${ }^{6}$ Keren et al confirmed the reliability of echocardiographic diagnosis of left ventricular bands in patients undergoing cardiac transplantation but cautioned that apical trabeculations could be misinterpreted as bands. ${ }^{18}$ In this study, when the McNemar analysis for paired data was repeated and any child with apical bands only $(n=4)$ was classified as having no bands, the prevalence of left ventricular bands in cases and controls was not significantly different.

It is widely accepted that innocent murmurs are less prevalent in older children and Harpin et al reported a temporal association between the disappearance of the left ventricular band and the murmur in one patient who was followed longitudinally. ${ }^{5}$ There are, however, no large longitudinal studies to provide supporting data for this observation and, indeed, a cross sectional study by Boyd et al found that the prevalence of left ventricular bands at necropsy was similar in each decade of life $(68 \%))^{19}$

We saw no turbulence about the bands during colour flow mapping, but we detected systolic curvilinear harmonic patterns in the left ventricle by pulsed wave Doppler, which were similar to those detected by Pennestri et al using continuous wave Doppler in hearts with structural abnormalities. ${ }^{2}$ They showed that murmurs can be produced by the vibration of intracardiac structures in the absence of turbulent flow. In our study these periodic vibrations and the audible systolic "whoops" were not confined to sites about the band, however, but could be clearly shown along the left ventricular walls. Although the relation of bands to murmurs was tenuous (see above), "whoops" were significantly more common in 
children with murmurs than in those without, suggesting that these appearances may be produced by vibration of the trabeculae lining the ventricular walls.

While the pulmonary peak flow and flow integrals did not differ significantly between cases and controls, the mean aortic peak flow, flow integral, and stroke volume were significantly greater in children with murmurs than in those without. These results support the observations of Schwarz et al that higher flow indices from the left side of the heart are seen in children with murmurs: but we could not confirm their finding that this is owing to the children with murmurs having smaller aortic diameters. ${ }^{7}$ It is unlikely that measurement of the aortic diameter at different levels (in the ascending aorta in their study and at the aortic valve in ours) accounted for this disparity. In contrast to the closely matched pairs of cases and controls in our study, the two groups in Schwarz et al's series were very different in terms of age and body surface area, though the difference in aortic diameter remained significant when corrected for body surface area.

Our study showed no difference in acceleration through the aortic valve between the cases and controls, nor did it seem that increased force of contraction was responsible for the murmur because the ejection fractions were similar in both groups. However, children with systolic murmurs had significantly lower heart rates than those without murmurs. Nearly all patients with very slow heart rates due to junctional bradycardia or complete heart block have a systolic murmur. It is possible that the prolonged diastolic filling of the left ventricle associated with a slower heart rate in the face of an unchanged ejection fraction and cardiac output, results in a higher stroke volume and aortic flow velocity and augments the intensity of the murmur. This explanation accords with the frequently observed finding that the innocent murmur becomes louder in the supine position-that is, when the heart rate naturally slows.

This study shows that there is a relation between vibratory phenomena within the left ventricle and the Still's vibratory murmur but the exact mechanism for its production requires further investigation and other factors may be implicated. The murmur seems to be augmented by increased flow volume and velocity and relative bradycardia; the role of left ventricular bands remains uncertain.
We thank our colleagues Dr S C Jordan and Dr R P Martin for referring suitable cases to us for this study and to Mrs J Evans and Mrs K Waters for their statistical advice.

McLaren MJ, Lachman AS, Pocock WA, Barlow JB. Innocent murmurs and third heart sounds in Black schoolchildren. Br Heart $J$ 1980;43:67-73.

2 Pennestri F, Boccardi L, Minardi G, Di Segni M, Pucci E Biasucci LM, et al. Doppler study of precordial musical murmurs. Am J Cardiol 1989;63:1390-4.

3 Sheikh MU, Lee WR, Mills RJ, Dais K. Musical murmurs: clinical implications, long-term prognosis, and echophonocardiographic features. Am Heart J 1984;108: phonocar

4 Stein PD, Sabbah HN, Milligan DJ, Lakier JB. Mechanism of a musical systolic murmur caused by a degenerated porcine $1874-82$.

5 Harpin VA, Ostman Smith I. The association of left ventricular tissue bands with vibratory innocent murmurs. Proceedings of the 59th annual meeting of the British Paediatric Association. Arch Dis Child 1987; 62:645-52.

6 Darazs B, Hesdorffer CS, Butterworth AM, Ziady F. The possible etiology of the vibratory systolic murmur. Clin Cardiol 1987;10:341-6.

7 Schwarz ML, Goldberg SJ, Wilson N, Allen HD, Marx GR Relation of Still's murmur, small aortic diameter and high aortic velocity. Am J Cardiol 1986;57:1344-8.

8 Sholler GF, Celermajer JM, Wright CM. Doppler echocardiographic assessment of cardiac output in normal children with and without innocent precordial murmurs. Am J Cardiol 1987;59:487-8.

9 Van Oort A, Van Dam I, Heringa A, Alsters J, Hopman J, Daniels $O$. The vibratory innocent heart murmur studied Daniels O. The vibratory innocent heart murmur studied by echo-d.

10 Brenner JI, Baker K, Ringel RE, Berman MA. Echocardiographic evidence of left ventricular bands in infants and children. J Am Coll Cardiol 1984;3:1515-20.

11 Chandraratua PA, Nanna M, McKay C, Nimalasuriaya A, Swinney R, Elkayam U, et al. Determination of cardiac output by transcutaneous continuous wave ultrasonic Doppler computer. Am J Cardiol 1984;53:234-7.

12 Marx GR, Goldberg SJ, Allen HD. Two methods for measurement of ascending aortic diameter by $2 \mathrm{D}$ echocardiography as compared with cineangiography. $\mathrm{Am}$ Heart $J 1986 ; 112: 172-3$.

13 Hudson I, Houston A, Aitchison T, Holland B, Turner T. Reproducibility of measurements of cardiac output in newborn infants by Doppler ultrasound. Arch Dis Child 1990;65:15-19.

14 Goldberg SJ, Sahn DJ, Allen HD, Valdez-Cruz LM, Hoenecke H, Carnahan Y. Evaluation of pulmonary and Hoenecke H, Carnahan Y. Evaluation of pulmonary and
systemic blood flow by two-dimensional Doppler echocardiography using fast Fourier transform spectral cardiography using fast Fourier trans
analysis. Am J Cardiol 1982;50:1394-1400.

15 Loeber CP, Goldberg SJ, Allen HD. Doppler echocardiographic comparison of flows distal to the four cardiac cardiographic comparison of flows distal to the
valves. J Am Coll Cardiol 1984;4:268-72.

16 Dickenson DF, Goldberg SJ, Wilson N. A comparison of information gained by cardiac catheterisation and ultrasound examination in pediatric patients with congenital heart disease. Int J Cardiol 1985;9:275-85.

17 Nishimura T, Kondo M, Shimada T, Shimono Y, Mukohyama N. Echocardiographic features of false tendons: with special reference to phonocardiographic significance. $J$ Cardiogr 1981;11:253-63.

18 Keren A, Billingham ME, Popp RL. Echocardiographic recognition and implications of ventricular hypertrophic trabeculations and aberrant bands. Circulation 1984, 70:836-42.

19 Boyd MT, Seward JB, Tajik AJ, Edwards WD. Frequency and location of prominent left ventricular trabeculations at autopsy in 474 normal human hearts: implications for autopsy in 474 normal human hearts: implications for evaluation of mural thrombi by two dimensiona
diography. J Am Coll Cardiol 1987;9:323-6. 